\title{
Duration and not strength of activation in temporo-parietal cortex positively correlates with schizotypy
}

\author{
Shahar Arzy, ${ }^{\mathrm{a}, \mathrm{b}}$ Christine Mohr, ${ }^{\mathrm{b}, \mathrm{c}}$ Christoph M. Michel, ${ }^{\mathrm{b}, \mathrm{d}}$ and Olaf Blanke ${ }^{\mathrm{a}, \mathrm{b}, *}$ \\ ${ }^{\mathrm{a}}$ Laboratory of Cognitive Neuroscience, Brain Mind Institute, Ecole Polytechnique Fédérale de Lausanne (EPFL), Lausanne, Switzerland \\ ${ }^{\mathrm{b}}$ Department of Neurology, University Hospital, Geneva, Switzerland \\ ${ }^{\mathrm{c}}$ Department of Experimental Psychology, University of Bristol, Bristol, UK \\ ${ }^{\mathrm{d} D e p a r t m e n t}$ of Fundamental Neuroscience, University Medical School, Geneva, Switzerland
}

Received 28 August 2006; revised 30 October 2006; accepted 16 November 2006

Available online 12 January 2007

Impaired self- and own body processing in patients with schizophrenia and individuals along the schizophrenia spectrum have been associated with dysfunctional cortical activation at the temporo-parietal junction. Here we investigated whether strength or duration of temporo-parietal junction activation during an own body processing task correlates with level of abnormal self-processing in healthy subjects as measured by the frequency of spontaneously experienced schizotypal body schema alterations (perceptual aberrations) and dissociative experiences. Participants carried out a mental imagery task with respect to their own body. Behavioral data and high density EEG were measured. EEG data were analyzed using evoked potential mapping and electrical neuroimaging. Participants completed two validated self-report questionnaires, one asking about perceptual aberration and one about dissociative experiences. The own body transformation task activated the right temporo-parietal junction at 310-390 ms. Participants' reaction times and duration of activation at the right temporo-parietal junction, but not its strength, were found to correlate positively with perceptual aberration scores. No relationship was found with dissociative experiences scores. Brain activations proceeding and following activation of the right temporo-parietal junction did not correlate with scores on either scale. The positive correlation between performance and right temporo-parietal activation in an own body transformation task with perceptual aberrations scores in our healthy population suggests that disturbances in self- and body processing in individuals along the schizophrenia spectrum might be due to prolonged, rather than stronger activation of the right temporo-parietal junction. We argue that this might reflect local pathology, pathologies in cortico-cortical connections and/or re-entry of top-down processing.

(C) 2006 Elsevier Inc. All rights reserved.

Keywords: Schizotypy; Embodiment; Self; Mental imagery; Perceptual aberrations; Temporo-parietal junction (TPJ)

\footnotetext{
* Corresponding author. Laboratory of Cognitive Neuroscience, Brain Mind Institute, Ecole Polytechnique Fédérale de Lausanne (EPFL), Station 15, 1015 Lausanne, Switzerland. Fax: +41 216939625.

E-mail address: olaf.blanke@epfl.ch (O. Blanke).

Available online on ScienceDirect (www.sciencedirect.com).
}

\section{Introduction}

Individuals along the schizophrenia spectrum - including patients with schizophrenia, individuals with a diagnosis of schizotypal personality disorder, and schizotypal individuals from the general healthy population - have been reported to suffer from several disturbances in self- and own body processing such as agency (Spence et al., 1997; Franck et al., 2002; Farrer et al., 2004; Lindner et al., 2005), self-other distinction (Platek and Gallup, 2002; Gallup et al., 2003; Farrer et al., 2004), theory of mind (Frith and Corcoran, 1996; Langdon and Coltheart, 1999), visual-perspective taking (Langdon and Coltheart, 2001; Mohr et al., 2006), and own body perception (Bleuler, 1911/1950; Angyal, 1936; Rado, 1960; Chapman et al., 1978; Meehl, 1990; Blakemore et al., 2000; Lenzenweger, 2000). Recent studies in healthy populations suggest that such self- and body processing relies on brain activity at the temporo-parietal junction (TPJ). The TPJ is activated during the visual processing of human movements (Beauchamp et al., 2002), visuo-spatial perspective taking (Maguire et al., 1998; Ruby and Decety, 2001; Vogeley and Fink, 2003), self-other distinction (Saxe and Kanwisher, 2003; Vogeley and Fink, 2003) and agency (Farrell and Robertson, 2000; Chaminade and Decety, 2002; Farrer and Frith, 2002), as well as mental own body imagery (Zacks et al., 1999; Blanke et al., 2005; Arzy et al., 2006a). Moreover, subjects with depersonalization disorder showed a higher activity in the TPJ, as measured by positron emission tomography (PET) (Simeon et al., 2000), and studies in neurological patients showed that damage to the TPJ may lead to disturbances in selfand body processing (Hécaen and Ajuriaguerra, 1952; Devinsky et al., 1989; Blanke et al., 2004; Blanke and Mohr, 2005; Arzy et al., 2006b).

Functional brain imaging studies testing self-processing in patients with schizophrenia found activation patterns at the TPJ that differed from those of other psychiatric patients and of healthy individuals. For example, using PET, Spence et al. (1997) examined patients with Schneiderian first-rank passivity symptoms 
of schizophrenia while they performed paced joystick movements and found that they showed stronger activation of the right TPJ (inferior parietal lobule) and cingulate gyrus compared to healthy subjects as well as patients with schizophrenia but without passivity symptoms. Notably, this increased activation diminished in these patients once symptoms disappeared. These results were replicated using functional magnetic resonance imaging (fMRI) (Ganesan et al., 2005). Franck et al. (2002) found a positive correlation between Schneiderian first-rank symptoms of schizophrenia and cerebral blood flow in right parietal cortex, as measured by PET. In a further study, Farrer et al. (2004) tested self-other discrimination with PET while healthy subjects and patients with Schneiderian first-rank symptoms of schizophrenia performed arm movements and watched differently distorted images of their arms displayed on a computer screen. Arm movements were presented to the participants with different degrees of angular distortions with respect to the actual arm movement that the participants were carrying out. In healthy individuals and patients, the right TPJ (angular gyrus) and the insula were activated. This activation was stronger in patients than healthy subjects and positively correlated with the presence of Schneiderian symptoms. Moreover, whereas the healthy individuals' activation at the right TPJ increased with increasing angular distortions, this was not the case for the patients. Collectively, these studies suggest that the right TPJ plays a central role in self- and body processing and that this processing is disturbed in patients with schizophrenia and neurological disorders with damage to this structure.

Recent studies (Blanke et al., 2005; Arzy et al., 2006a) have reported selective TPJ activation in self- and body-processing tasks when healthy subjects mentally changed their body position and spatial perspective with respect to their own body (own body transformation task, OBT-task). Furthermore, Mohr et al. (2006) showed that performance in this OBT-task positively correlated with degree of positive schizotypy as measured by the frequency of spontaneously experienced schizotypal perceptual alterations (perceptual aberration score; PA score; (Chapman et al., 1978)). As high PA scores have been considered an indicator of psychosis-proneness (Chapman et al., 1994; Lenzenweger, 1994; Tallent and Gooding, 1999), these findings would suggest that positive schizotypy (and by inference passivity symptoms in schizophrenia) might also be associated with impaired neural processing at the TPJ, in concordance with findings in patients with schizophrenia (Spence et al., 1997; Franck et al., 2002; Farrer et al., 2004). Yet, due to their limited temporal resolution the abovementioned fMRI and PET studies did not allow to distinguish between strength-related versus duration-related increases in TPJ activation and can thus not assess some of the basic mechanisms of the functional aberrations in schizophrenic patients (Lehmann et al., 2005). Therefore, to further study the link between TPJ activation in positive schizotypy, we here used evoked potential (EP) mapping and electrical neuroimaging with temporal resolution in the millisecond range (Gevins et al., 1995; Dale and Halgren, 2001; Michel et al., 2001; Koenig et al., 2005; for the use of this method in patients with schizophrenia see Miyauchi et al., 1990; John et al., 1994; Omori et al., 1995; Saito et al., 1998; Koenig et al., 1999; Lehmann et al., 2005) to investigate whether not only performance but also brain activity in the OBT-task correlates with PA scores.

\section{Methods}

Participants

Thirteen healthy male volunteers (aged 19-31 years, mean \pm SD: $25.0 \pm 3.2$ years) participated in the study. All participants were right-handed according to the Edinburgh handedness inventory (Oldfield, 1971), had normal or corrected-to-normal vision, and no history of neurological or psychiatric disorders. All participants gave written informed consent prior to inclusion in the study, which had been approved by the Ethical Committee of the University Hospital of Geneva (Switzerland).

\section{Stimuli}

Participants were presented with a set of four visual stimuli: a human figure, which could be facing toward or away from the participant (front- vs. back-facing) and which had one hand marked such that it appeared as wearing a gray glove and a black ring at the wrist (Fig. 1). This indication of side could appear on the right or on the left hand (Zacks et al., 1999; Blanke et al., 2005; Arzy et al., 2006a). Front- and back-facing figures had the same dimensions $\left(5.0 \times 6.1^{\circ}\right.$ of visual angle $)$ and differed only in the rendering of the clothing of the figure and the presence of a face or the back of a head. Stimuli appeared for $200 \mathrm{~ms}$ in the center of the computer screen with an inter-stimulus interval of $2000 \mathrm{~ms}$.

\section{Procedure}

In the own body transformation task (OBT-task), participants were asked to make right-left judgments while imagining themselves in the body position of the figure, taking its visuospatial perspective, thus involving mental own body positioning (Blanke et al., 2005; Arzy et al., 2006a). Left-right judgments were given via a button press on a serial response box. Responses were given using index and middle fingers of the left and right hand in alternating blocks. The participants were instructed to respond as fast and precisely as possible but always to perform the requested mental imagery before giving the response. The experiment included 4 blocks, each included a total of 160 trials, which consisted of 40 presentations of each stimulus in a randomized order. We calculated mean reaction times for correct responses.

\section{Questionnaires}

After the experiment, participants completed the standardized handedness questionnaire (Oldfield, 1971) and the 35-item true-
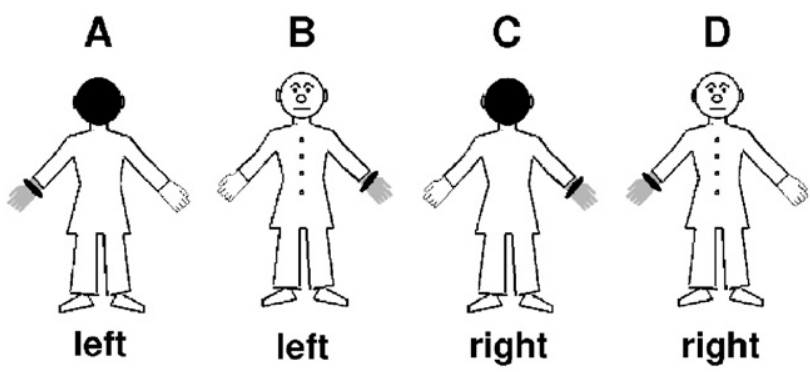

Fig. 1. Stimuli. The four different stimuli as used in the own body transformation (OBT) task are shown. Correct responses are indicated below each figure. 
false self-report PA scale (Chapman et al., 1978). Typical items of this latter scale are "Occasionally I have felt as though my body did not exist" and "Occasionally I have the impression that one of my body-parts is bigger than usual" (Chapman et al., 1994; Lenzenweger, 1994; Tallent and Gooding, 1999). Participants completed also the 28-item dissociative experiences (DE) scale (Carlson et al., 1993), which was developed for measuring the frequency of dissociative experiences. Typical items of this scale are "Some people have the experience of finding themselves in a place and have no idea how they got there" and "Some people find that sometimes they are listening to someone talk and they suddenly realize that they did not hear part or all of what was said". Participants had to scale what percentage of the time such experiences happened to them. In addition, participants were asked about their previous neurological or psychiatric history. Since the DE scale is positively correlated with the PA scale (Pope and Kwapil, 2000), and DEs reflect disturbed self-processing, we additionally took this questionnaire into account (Mohr and Blanke, 2005).

\section{Analysis of behavioral data}

Repeated measures ANOVAs were run on reaction times and accuracy with the orientation of body stimuli (back-facing vs. front-facing) as the repeated measures factor. Then, to test possible relationships between individuals' questionnaire scores and task performance, separate correlation analyses were performed between reaction times and accuracy for the different task conditions (front-facing, back-facing, and mean) and the different questionnaire scores (PA and DE). These were performed for the whole sample, using Pearson product moment correlations. All $p$-values are two-tailed, and the significance level was set to $\alpha=0.05$.

\section{Electroencephalography (EEG) recording and evoked potential (EP) mapping}

Continuous EEG was acquired with a Geodesics Netamps system (Electrical Geodesics, Inc., USA) from 123 scalp electrodes (impedances $<50 \mathrm{k} \Omega$; vertex referenced; $500-\mathrm{Hz}$ digitization; band-pass filtered $0.1-200 \mathrm{~Hz}$ ) in a darkened, electrically shielded booth. Epochs of EEG (from 0 to $600 \mathrm{~ms}$ post-stimulus onset) from trials yielding correct responses were averaged for each of the two experimental conditions and for each subject to calculate the EPs (Lehmann, 1987). In addition to the rejection of sweeps where any channel exceeded the amplitude of $\pm 100 \mu \mathrm{V}$, the data were visually inspected to reject epochs with blinks, eye movements, or other sources of transient noise. For each subject's EPs, the electrodes on the outermost circumference (chin and neck) as well as other artefact channels were excluded and interpolated to a standard 111-channel electrode array (twodimensional spherical spline; Perrin et al., 1987). After this procedure and before group averaging, EPs were band-pass filtered $(1-40 \mathrm{~Hz})$ and recalculated against the average reference (Lehmann, 1987).

The 111-channel EP analysis was based on the examination of the spatial variations of the voltage distribution over time and between conditions, an approach known as microstates EP mapping (Lehmann and Skrandies, 1984; Brandeis and Lehmann, 1986; Michel et al., 1999, 2001). This approach searches for time segments of stable map topography that represent functional microstates of the brain during information processing. EP microstate segments were defined by using a spatial $k$-means cluster analysis to identify the dominant map topographies in the group-averaged evoked potentials across the experimental conditions over time (Pascual-Marqui et al., 1995; Michel et al., 2001, 2004). The optimal number of these template maps is determined by a modified cross-validation criterion (Pascual-Marqui et al., 1995; Michel et al., 2001, 2004). In a second step the presence of a given EP map as identified in the group-averaged data was verified statistically in the EPs of the individual subjects. EP maps were competitively fitted to the EPs of the individual subjects. This allows to determine the duration (number of time-points that were assigned to one microstate map) and the amplitude (or global field power, GFP) of a given EP map for each condition across subjects. These duration and GFP values for a given EP map then can be subjected to statistical analysis. Statistical comparisons were performed on the duration and GFP of each map (dependent variable) in the individual EPs using repeated measures ANOVAs, with the orientation of body stimuli (backfacing vs. front-facing) as the repeated measures factor. Then, to test possible relationships between individuals' questionnaire scores and the measured brain activity, the respective mean durations and GFP of the different EP maps for the individual subjects were correlated with participants' questionnaire scores. For each EP map, separate correlation analyses were performed (for duration and GFP) in the different task conditions (frontfacing, back-facing, and mean) and both questionnaire scores (PA and DE). These were performed for the whole sample, using Pearson product moment correlations. All $p$-values are two-tailed, and the significance level was set to $\alpha=0.05$.

\section{Source localization}

The neural generators for a given mean EP map were estimated by using a distributed linear inverse solution, based on a local auto-regressive average (LAURA) model (Grave de Peralta et al., 2004) as used previously (Blanke et al., 2005; Arzy et al., 2006a). LAURA selects the source configuration that tries to mimic the biophysical behavior of electric vector fields (i.e., activity at one point depends on the activity at neighboring points according to electromagnetic laws). The solution space was calculated on a realistic head model that included 4024 nodes, selected from a $6 \times 6 \times 6 \mathrm{~mm}$-grid equally distributed within the gray matter of the Montreal Neurological Institute's average brain.

\section{Results}

\section{Task performance and questionnaire scores}

Reaction times were found to be significantly longer for frontfacing (mean \pm SD: $805.8 \pm 95.3 \mathrm{~ms}$ ) than back-facing $(696.8 \pm$ $68.8 \mathrm{~ms})$ figures $\left(F_{(1,12)}=58.3 p<0.001\right)$, reflecting results of previous studies (Zacks et al., 1999; Blanke et al., 2005; Arzy et al., 2006a; Mohr et al., 2006). Mean PA (5.2 \pm 2.4$)$ and DE (10.3 \pm 7.6) scores were comparable to those reported in previous studies (6.6 \pm 6.0 ; (Chapman et al., 1978) and 10.8 7.1 (Carlson et al., 1993), respectively).

PA scores correlated positively with reaction times across task conditions in the OBT-task ( $r=0.70 ; p<0.01$; Fig. 2A). In addition, the correlation was higher in the front condition $(r=0.73 ; p<0.01)$ 
A

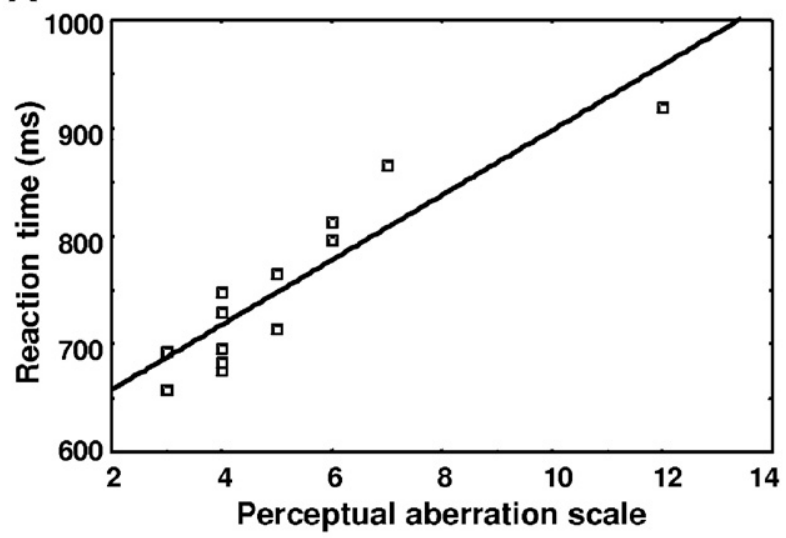

B

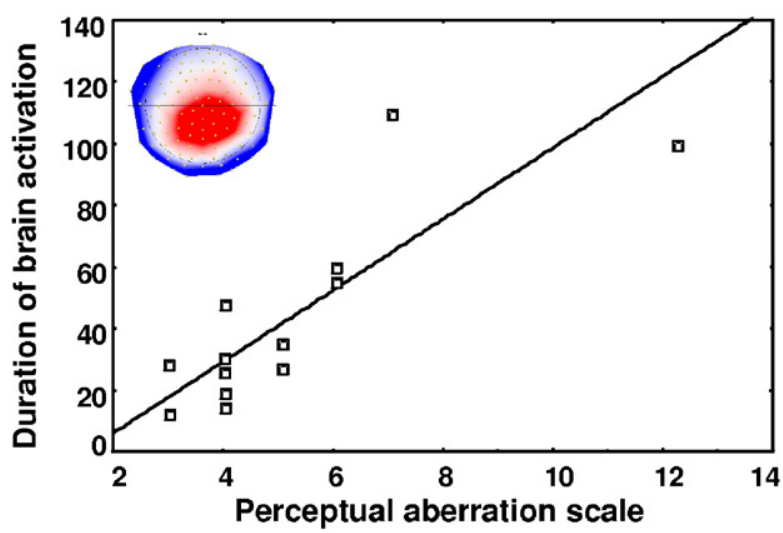

Fig. 2. Correlations between reaction times, brain activation, and perceptual aberrations (PA) scores. (A) Reaction times in the OBT-task as a function of individuals' PA scores. (B) Electric brain topography during the OBT-task (OBT map) is shown. Duration of OBT map, representing brain activation, is plotted as a function of individuals' PA scores. Note that this correlation parallels to the correlation between the behavioral results and the PA scores.

than in the back condition $(r=0.60 ; p<0.05)$. These correlations were absent when reaction times were compared with the DE scores (all $r$-values $<0.23$; all $p$-values $>0.44$; Fig. $3 \mathrm{~A}$ ). PA and DE scales were not correlated to each other $(r=0.17 ; p=0.61)$. With regard to accuracy, participants' error rates were higher in the front condition than in back condition (mean error rates: $12.9 \pm 10.9 \%$ (front); $7.2 \pm 7.5 \%$ (back); $F_{(1,12)}=7.85, p<0.05$ ). Accuracy rates did neither correlate with PA scores $(r=0.12 ; p=0.71)$ nor with DE scores $(r=0.51 ; p=0.18)$.

\section{EP mapping and source localization}

EP mapping of the group-averaged data revealed one microstate of brain activation (time segment of stable voltage topography; EP map) that lasted significantly longer for the front-facing than the back-facing figures $\left(F_{(1,12)}=6.4, p=0.05\right.$; Fig. 4A, B), suggesting that this EP map is linked to OBT as reported previously (Blanke et al., 2005; Arzy et al., 2006a). This microstate, as reflected by the OBT map, lasted from 310 to $390 \mathrm{~ms}$ (earliest and latest appearance of the OBT map in front and back conditions). These statistical differences in duration were not found for other EP maps before and after the OBT map (see Supplementary Table 1 online).
No difference was found when statistical analysis was performed on the amplitude (or GFP) of those EP maps (see Supplementary Table 1 online). A linear inverse solution (LAURA; Grave de Peralta et al., 2004) localized the OBT map to the right TPJ (maximal peak of Talairach coordinates: $52-63$ 8) and left lateral occipito-temporal cortex (maximal peak: $-49-68$ 1; Fig. 4C), confirming previous results in different study samples (Blanke et al., 2005; Arzy et al., 2006a).

\section{Questionnaire scores and TPJ activation}

Comparison of PA scores with the duration of the OBT map for each subject revealed a significant positive correlation $(r=0.69$; $p<0.01$; Fig. 2B). No other EP map, before and after the OBT map, showed a significant correlation with PA scores (see Supplementary Table 1 online). Comparable to the relationship between reaction times and PA scores, the correlation between TPJ activation and PA scores was more pronounced for front-facing figures $(r=0.77 ; p<0.001)$ than for back-facing figures $(r=0.51$; $p=0.07)$. In addition, comparable correlations between the duration of the OBT map were unrelated to DE scores (Fig. 3B; see Supplementary Table 1 online). Finally, neither for PA scores nor for DE scores were significant correlations found with the strength (or GFP) of the different EP maps (see Supplementary Table 1
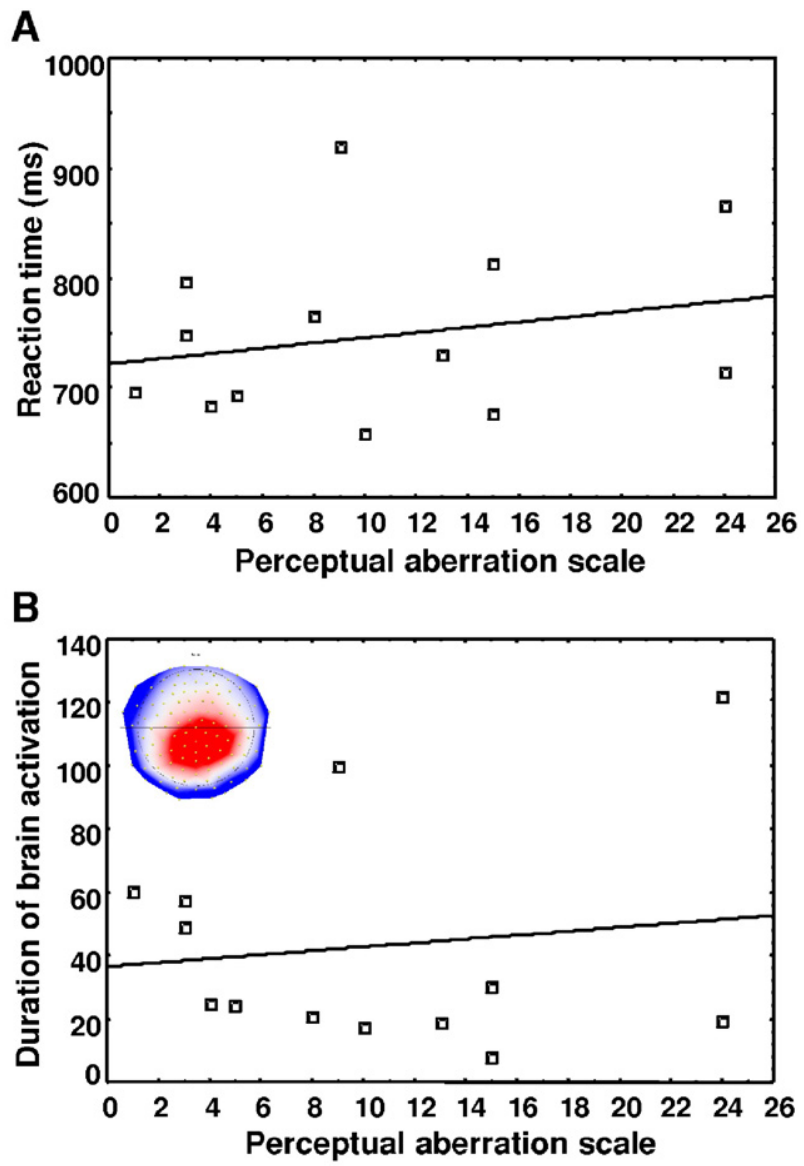

Fig. 3. Correlations between reaction times, brain activation and dissociative experience (DE) scores. (A) Reaction times in the OBT-task as a function of individuals' DE scores. (B) Duration of OBT map, representing brain activation, is plotted as a function of individuals' DE scores. 
A $[\mu \mathrm{V}]$
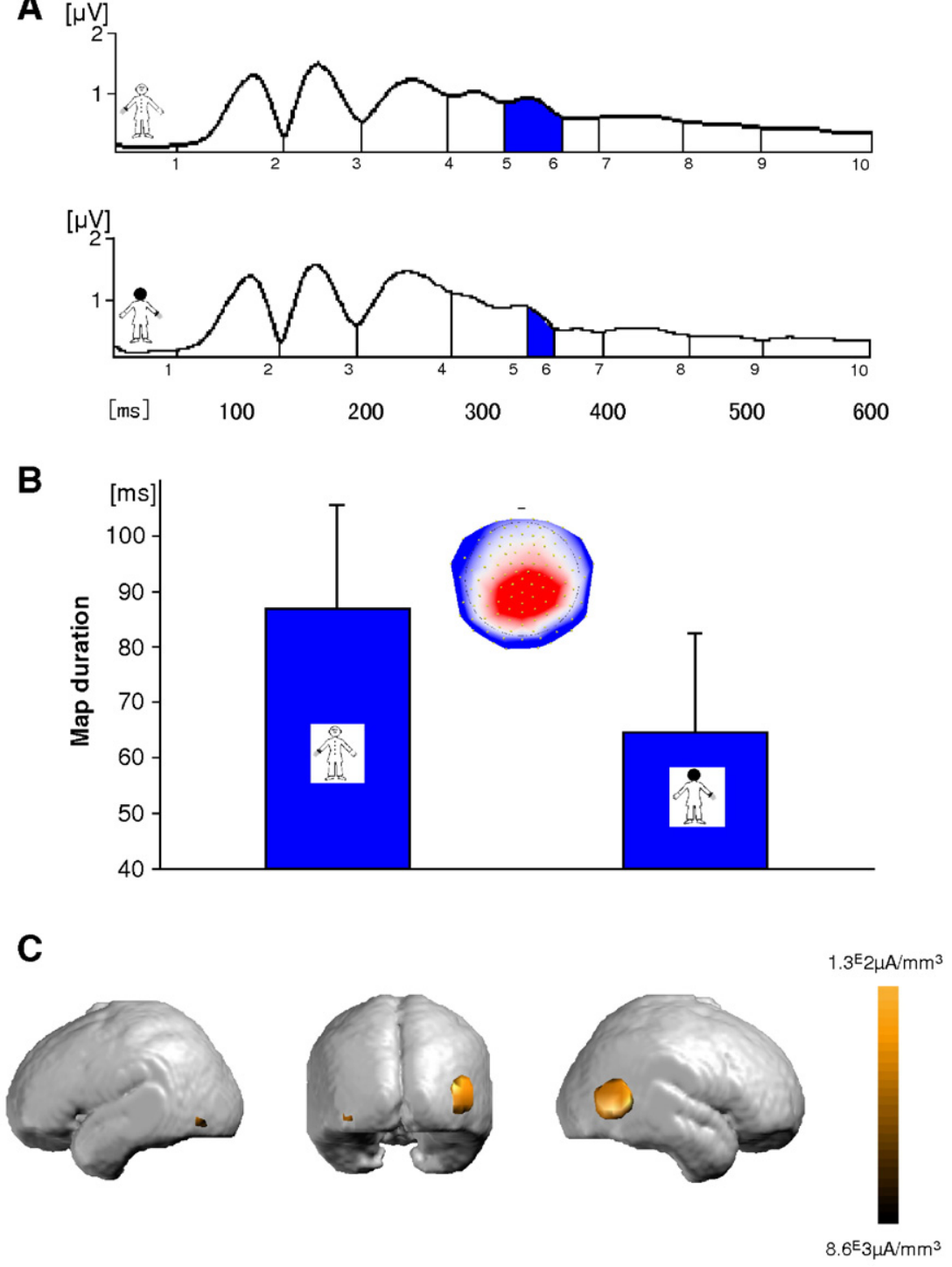

Fig. 4. Evoked potential (EP) data during task performance. (A) Segments of stable map topography in the two experimental conditions under the global field power curve from 0 to $600 \mathrm{~ms}$. EP segment 6 (OBT map; segment shown in blue) was found from 310 to $390 \mathrm{~ms}$ and was longer for the front-facing figures than to the back-facing figures. (B) Duration of OBT map for the two experimental conditions for all participants. (C) Generators of OBT map were localized at the right TPJ (maximal peak of Talairach coordinates: $52-63$ 8) and left lateral occipito-temporal cortex (maximal peak: $-49-68$ 1).

online). These data suggest that the duration of TPJ activation during the OBT-task, and not its amplitude, in a specific time period ( 310-390 ms after stimulus onset), is positively correlated with positive schizotypy (PAs). No such meaningful relationship was found for DE scores.

To summarize, our data show [1] that activation of the right TPJ is involved in self- and body-processing and is prolonged as a function of positive schizotypy in the general healthy population. In addition, our results were [2] selective for the activation of the TPJ at $310-390 \mathrm{~ms}$ as prior or later activations were unrelated to participants' positive schizotypal experiences. Finally, our results using electrical neuroimaging [3] extend previous neuroimaging findings (Spence et al., 1997; Franck et al., 2002; Farrer et al., 2003, 2004; Ganesan et al., 2005) in showing that the duration and not the strength of TPJ activation is related to disorders along the schizophrenia spectrum (see Discussion).

\section{Discussion}

\section{Self-processing, schizotypy, and TPJ activation}

The present data confirm in an independent study sample that performance in the OBT-task positively correlates with the degree of schizotypy as reflected by PA scores (Mohr et al., 2006), but not dissociation as measured by the DE scores. This observation is in line with other behavioral evidence showing that self- and body processing are impaired along the schizophrenia spectrum (Bleuler, 1911/1950; Chapman et al., 1978; Frith and Corcoran, 1996; Blakemore et al., 2000; Lenzenweger, 2000; Langdon and Coltheart, 2001; Franck et al., 2002; Platek and Gallup, 2002; Gallup et al., 2003; Farrer et al., 2004; Lindner et al., 2005). With respect to schizotypy, previous studies have suggested that positive schizotypy relates to experimentally induced own body distortions 
(Burrack and Brugger, 2005) as well as to relatively impaired performance in egocentric visuo-spatial perspective taking (Langdon and Coltheart, 2001). Our behavioral data suggest that healthy participants scoring higher on positive schizotypy are relatively more impaired in mental imagery with respect to their own body (Mohr et al., 2006). Our EP data also confirm that the OBT-task selectively activates the right TPJ at 310-390 ms (Blanke et al., 2005; Arzy et al., 2006a) and that this activation positively correlates with behavioral measures. This suggests that the above impairment in own body imagery in our healthy participants scoring high on positive schizotypy is related to impaired processing at the right TPJ. This would also concord with the finding that schizotypy may be characterized by experiences of disembodiment (American Psychiatric Association, 2000), which are linked to mental own body imagery and the TPJ (Blanke et al., $2002,2005)$. Increases in reaction time ( $200 \mathrm{~ms})$ were larger than increase in duration of the OBT map $(\sim 100 \mathrm{~ms})$. This indicates that other processes might also be delayed in individuals with higher PA scores. Finally, our data also corroborate previous EP data showing that patients with schizophrenia suffer from prominent processing deficits around $300 \mathrm{~ms}$ after stimulus onset. Yet, this was related to changes of the P300 component and measured during different behavioral paradigms (such as the oddball paradigm) whereas we used a mental transformation task in the present study (Roth and Cannon, 1972; Roth et al., 1980; Strik et al., 1994; Heidrich and Strik, 1997).

\section{Duration and not strength of right TPJ activation correlates with schizotypy}

The present study shows that degree of schizotypy in healthy subjects, reflected by PAs, positively correlates with the duration of the OBT map, reflecting activation at the right TPJ. Individuals with higher PA scores thus activated the right TPJ for a longer time and took longer for responding. This is similar to previous studies in patients with schizophrenia where activity at the right TPJ was positively correlated with Schneiderian scores (Franck et al., 2002; Farrer et al., 2004) pointing to similar processes in individuals along the schizophrenia spectrum (see below). Correlation between degree of schizotypy and duration of activation at the right TPJ was found in the present study only for the time period between 310 and $390 \mathrm{~ms}$ and was absent for earlier and later brain activations. Moreover, this correlation was statistically stronger in the frontfacing than back-facing condition, with the former being also more demanding than the back-facing condition as reflected in reaction times and duration of the TPJ activation.

TPJ activity related to self-processing has been shown to be impaired in patients with schizophrenia (Frith, 1996; Frith and Dolan, 1996; Spence et al., 1997; Franck et al., 2002; Farrer et al., 2004; Ganesan et al., 2005; see Introduction). However, whereas earlier studies suggested that pathological TPJ activity was due to changes in strength of activation, the present data suggest that increased TPJ activity was due to longer, but not stronger, TPJ activation. In addition, this longer activation was only found for a selective time period. Based on the present data, we speculate that previously observed pathological changes in right TPJ activation in patients with schizophrenia (Spence et al., 1997; Franck et al., 2002; Farrer et al., 2004; Ganesan et al., 2005) were also due to abnormally prolonged TPJ activation rather than abnormal increases in activation (as neuroimaging data reported in these studies do not allow to distinguish between both possibilities). Yet, comparison between the present study and former studies in patients with schizophrenia needs to be regarded with caution for several reasons. First, different self-related tasks have been used in the different studies (no task: Franck et al., 2002; own hand movement: Spence et al., 1997; Farrer et al., 2004; Ganesan et al., 2005; own body transformation: present study) making comparison across studies difficult. Second, we here tested participants from the general population, while the previous studies tested patients with schizophrenia, who were also treated with neuroleptic medication, likely affecting their performance and brain activation. Third, it might be the case that schizotypal individuals from the general population and patients with schizophrenia might differ in their deficits in self- and body processing and the underlying neural mechanisms. Such a difference would be in line with notions of discontinuities along the schizophrenia spectrum (Siever and Davis, 2004; Mohr et al., 2005). Finally, different brain imaging techniques have been used in the different studies, again making comparison across studies difficult. Nevertheless, the data from these studies using different populations along the schizophrenia spectrum, different self tasks, and different neuroimaging methods point to a key role of the right TPJ in self- and body processing in schizophrenia.

The present data show that increased TPJ activity was due to longer, but not stronger, TPJ activation during a selective time period. Several neural mechanisms have been proposed to account for prolonged brain activation patterns. David et al. (2005) proposed that increases in duration of activation may depend on increased backward (top-down) connections, reflecting re-entry of neural signals to lower-tier processing areas as EPs were found to be more enduring and dispersed in higher level areas (David et al., 2005). This is particularly true with respect to late EP components (David et al., 2005), like the OBT map, which we found for the time period between 310 and $390 \mathrm{~ms}$. The prolonged activity found in the present study might thus reflect abnormal processing at the right TPJ related to such altered top-down signals in subjects with high schizotypy scores. Alternatively, prolongation of activation at the right TPJ may also be due to an increase of independent simultaneous brain processes and/or degrading of functional connectivity (Friston and Frith, 1995; Friston, 1996; Saito et al., 1998). However, as a stable map across time indicates that the same brain generators are active and functionally connected across this time period (Lehmann, 1987), it is unlikely that other brain processes or degradation in connectivity would prolong a stable topographic map. In the latter condition one would rather predict a change in map topography.

At last, contrary to our predictions (Mohr and Blanke, 2005), no correlation was observed between the degree of dissociation (as measured by DE scores) and behavioral reaction times or brain activation at the TPJ during the OBT-task. Explanation of this latter observation remains unclear at this point. For instance, previous studies found DEs to be related to PAs (Pope and Kwapil, 2000) and positive schizotypy in a more general sense (Irwin, 2000). Also disturbed self-processing such as experienced during out-of-body experiences has been linked to dissociative experiences (Murray and Fox, 2005), albeit in the latter case this relationship has been made with respect to somatoform DEs and not with respect to the presently used DE scale. Thus, future studies have to further evaluate the relevance of DEs to impaired own body processing as well as self-processing in addition to a presumed link with the TPJ. 
In conclusion, the present study shows that the right TPJ activation in the specific time window of $\sim 310-390 \mathrm{~ms}$ as well as performance measures in an OBT-task are positively correlated with degree of schizotypy (as measured by PA scores). Our electrical neuroimaging data suggest that the degree of schizotypy positively correlated with the duration and not the strength of right TPJ activation. Pathological activations in previous neuroimaging studies in healthy subjects and patients with schizophrenia (using fMRI and PET) at the right TPJ might thus be due rather to increased duration than strength of activation at this site, pointing to different neural mechanisms. The present data suggest that, due to prolonged and relative impaired activation of the right TPJ, individuals with increased levels of schizotypy are relatively impaired in self-processing. We suggest that disturbances of selfand body processing in psychiatric and neurological patients as well as individuals along the schizophrenia spectrum share neural mechanisms in this brain region.

\section{Acknowledgments}

Supported by Fondation de Famille Sandoz and by the Centre d'Imagerie BioMedicale (CIBM) of Geneva and Lausanne, Switzerland. S.A. is supported by the Isaiah Horowitz Foundation via the Center for Complexity Science.

\section{Appendix A. Supplementary data}

Supplementary data associated with this article can be found, in the online version, at doi:10.1016/j.neuroimage.2006.11.027.

\section{References}

Angyal, A., 1936. The experience of the body-self in schizophrenia. Arch. Neurol. Psychiatry 35, 1029-1053.

Arzy, S., Thut, G., Mohr, C., Michel, C.M., Blanke, O., 2006a. Neural basis of embodiment: distinct contribution of temporoparietal junction and extrastriate body area. J. Neurosci. 26, 8074-8081.

Arzy, S., Seeck, M., Ortigue, S., Spinelli, L., Blanke, O., 2006b. Induction of an illusory shadow person. Nature 443, 287.

American Psychiatric Association, 2000. Diagnostic and Statistical Manual of Mental Disorders, 4th ed. American Psychiatric Press, Washington, DC.

Beauchamp, M.S., Lee, K.E., Haxby, J.V., Martin, A., 2002. Parallel visual motion processing streams for manipulable objects and human movements. Neuron 34, 149-159.

Blakemore, S.-J., Smith, J., Steel, R., Johnstone, C.E., Frith, C.D., 2000. The perception of self-produced sensory stimuli in patients with auditory hallucinations and passivity experiences: evidence for a breakdown in self-monitoring. Psychol. Med. 30, 1131-1139.

Blanke, O., Mohr, C., 2005. Out-of-body experience, heautoscopy, and autoscopic hallucination of neurological origin. Implications for neurocognitive mechanisms of corporeal awareness and self-consciousness. Brain Res. Brain Res. Rev. 50, 184-199.

Blanke, O., Ortigue, S., Landis, T., Seeck, M., 2002. Stimulating illusory own-body perceptions. Nature 419, 269-270.

Blanke, O., Landis, T., Spinelli, L., Seeck, M., 2004. Out-of-body experience and autoscopy of neurological origin. Brain 127, 243-258.

Blanke, O., Mohr, C., Michel, C.M., Pascual-Leone, A., Landis, T., Thut, G., 2005. Linking out-of-body experience and self processing to mental own-body imagery at the temporoparietal junction. J. Neurosci. 25, $550-557$.
Bleuler, E., 1911/1950. Dementia Praecox or the Group of the Schizophrenias. International Univ. Press, New York.

Brandeis, D., Lehmann, D., 1986. Event-related potentials of the brain and cognitive processes: approaches and applications. Neuropsychologia 24 , $151-168$.

Burrack, A., Brugger, P., 2005. Individual differences in susceptibility to experimentally induced phantom sensations. Body Image 2, 307-313.

Carlson, E.B., Putnam, F.W., Ross, C.A., Torem, M., Coons, P., Dill, D.L., Loewenstein, R.J., Braun, B.G., 1993. Validity of the Dissociative Experiences Scale in screening for multiple personality disorder: a multicenter study. Am. J. Psychiatry 150, 1030-1036.

Chaminade, T., Decety, J., 2002. Leader or follower. Involvement of the inferior parietal lobule in agency? NeuroReport 13, 1975-1978.

Chapman, L.J., Chapman, J.P., Raulin, M.L., 1978. Body-image aberration in schizophrenia. J. Abnorm. Psychol. 87, 399-407.

Chapman, L.J., Chapman, J.P., Kwapil, T.R., Eckblad, M., Zinser, M.C., 1994. Putatively psychosis-prone subjects 10 years later. J. Abnorm. Psychol. 103, 171-183.

Dale, A.M., Halgren, E., 2001. Spatiotemporal mapping of brain activity by integration of multiple imaging modalities. Curr. Opin. Neurobiol. 11, 202-208.

David, O., Harrison, L., Friston, K.J., 2005. Modelling event-related responses in the brain. NeuroImage 25, 756-770.

Devinsky, O., Feldmann, E., Burrowes, K., Bromfield, E., 1989. Autoscopic phenomena with seizures. Arch. Neurol. 46, 1080-1088.

Farrell, M.J., Robertson, I.H., 2000. The automatic updating of egocentric spatial relationships and its impairment due to right posterior cortical lesions. Neuropsychologia 38, 585-595.

Farrer, C., Frith, C.D., 2002. Experiencing oneself vs. another person as being the cause of an action: the neural correlates of the experience of agency. NeuroImage 15, 596-603.

Farrer, C., Franck, N., Georgieff, N., Frith, C.D., Decety, J., Jeannerod, M., 2003. Modulating the experience of agency: a positron emission tomography study. NeuroImage 18, 324-333.

Farrer, C., Franck, N., Frith, C.D., Decety, J., Georgieff, N., d'Amato, T., Jeannerod, M., 2004. Neural correlates of action attribution in schizophrenia. Psychiatry Res. 131, 31-44.

Franck, N., O'Leary, D.S., Flaum, M., Hichwa, R.D., Andreasen, N.C., 2002. Cerebral blood flow changes associated with Schneiderian firstrank symptoms in schizophrenia. J. Neuropsychiatry Clin. Neurosci. 14, 277-282.

Friston, K.J., 1996. Theoretical neurobiology and schizophrenia. Br. Med. Bull. 52, 644-655.

Friston, K.J., Frith, C.D., 1995. Schizophrenia: a disconnection syndrome? Clin. Neurosci. 3, 89-97.

Frith, C., 1996. Neuropsychology of schizophrenia: what are the implications of intellectual and experiential abnormalities for the neurobiology of schizophrenia? Br. Med. Bull. 52, 618-626.

Frith, C.D., Corcoran, R., 1996. Exploring 'theory of mind' in people with schizophrenia. Psychol. Med. 26, 521-530.

Frith, C., Dolan, R., 1996. The role of the prefrontal cortex in higher cognitive functions. Brain Res. Cogn. Brain Res. 5, 175-181.

Gallup Jr., G.G., Anderson, J.R., Platek, S.M., 2003. Self-awareness, social intelligence, and schizophrenia. In: Kircher, T.T., David, S. (Eds.), The Self and Schizophrenia: A Neuropsychological Perspective. Cambridge Univ. Press, Cambridge, UK.

Ganesan, V., Hunter, M.D., Spence, S.A., 2005. Schneiderian first-rank symptoms and right parietal hyperactivation: a replication using FMRI. Am. J. Psychiatry 162, 1545.

Gevins, A.S., Leong, H., Smith, M.E., Le, J., Du, R., 1995. Mapping cognitive brain function with modern high-resolution electroencephalography. Trends Neurosci. 18, 429-436.

Grave de Peralta, R., Murray, M.M., Michel, C.M., Martuzzi, R., Gonzalez Andino, S.L., 2004. Electrical neuroimaging based on biophysical constraints. NeuroImage 21, 527-539.

Hécaen, H., Ajuriaguerra, J., 1952. L'Heautoscopie, Meconnassiances et Hallucinations Corporelles. Masson, Paris. 
Heidrich, A., Strik, W.K., 1997. Auditory P300 topography and neuropsychological test performance: evidence for left hemispheric dysfunction in schizophrenia. Biol. Psychiatry 41, 327-335.

Irwin, H.J., 2000. The disembodied self: an empirical study of dissociation and the out-of-body experience. J. Parapsychol. 64, 261-276.

John, E.R., Prichep, L.S., Alper, K.R., Mas, F.G., Cancro, R., Easton, P., Sverdlov, L., 1994. Quantitative electrophysiological characteristics and subtyping of schizophrenia. Biol. Psychiatry 36, 801-826.

Koenig, T., Lehmann, D., Merlo, M.C., Kochi, K., Hell, D., Koukkou, M., 1999. A deviant EEG brain microstate in acute, neuroleptic-naive schizophrenics at rest. Eur. Arch. Psychiatry Clin. Neurosci. 249, 205-211.

Koenig, T., Studer, D., Hubl, D., Melie, L., Strik, W.K., 2005. Brain connectivity at different time-scales measured with EEG. Philos. Trans. R. Soc., B 360, 1015-1023.

Langdon, R., Coltheart, M., 1999. Mentalising, schizotypy, and schizophrenia. Cognition 71, 43-71.

Langdon, R., Coltheart, M., 2001. Visual perspective-taking and schizotypy: evidence for a simulation-based account of mentalizing in normal adults. Cognition 82, 1-26.

Lehmann, D., 1987. Principles of spatial analysis. In: Gevins, A.S., Remond, A. (Eds.), Methods of Analysis of Brain Electrical and Magnetic Signals. Handbook of Electroencephalography and Clinical Neurophysiology, vol. 1. Elsevier, Amsterdam, pp. 309-354.

Lehmann, D., Skrandies, W., 1984. Spatial analysis of evoked potentials in man-A review. Prog. Neurobiol. 23, 227-250.

Lehmann, D., Faber, P.L., Galderisi, S., Herrmann, W.M., Kinoshita, T., Koukkou, M., Mucci, A., Pascual-Marqui, R.D., Saito, N., Wackermann, J., Winterer, G., Koenig, T., 2005. EEG microstate duration and syntax in acute, medication-naive, first-episode schizophrenia; a multi-center study. Psychiatry Res. 138, 141-156.

Lenzenweger, M.F., 1994. Psychometric high-risk paradigm, perceptual aberrations, and schizotypy: an update. Schizophr. Bull. 20, 121-135.

Lenzenweger, M.F., 2000. Two-point discrimination thresholds and schizotypy: illuminating a somatosensory dysfunction. Schizophr. Res. 42, 111-124.

Lindner, A., Thier, P., Kircher, T.T., Haarmeier, T., Leube, D.T., 2005. Disorders of agency in schizophrenia correlate with an inability to compensate for the sensory consequences of actions. Curr. Biol. 15, 1119-1124.

Maguire, E.A., Burgess, N., Donnett, J.G., Frackowiak, R.S., Frith, C.D., O'Keefe, J., 1998. Knowing where and getting there: a human navigation network. Science 280, 921-924.

Meehl, P.E., 1990. Toward an integrated theory of schizotaxia, schizotypy and schizophrenia. J. Pers. Disord. 4, 1-99.

Michel, C.M., Seeck, M., Landis, T., 1999. Spatio-temporal dynamics of human cognition. News Physiol. Sci. 14, 206-214.

Michel, C.M., Thut, G., Morand, S., Khateb, A., Pegna, A.J., Grave de Peralta, R., Gonzalez, S., Seeck, M., Landis, T., 2001. Electric source imaging of human brain functions. Brain Res. Brain Res. Rev. 36, 108-118.

Michel, C.M., Murray, M.M., Lantz, G., Gonzalez, S., Spinelli, L., Grave de Peralta, R., 2004. EEG source imaging. Clin. Neurophysiol. 115, $2195-2222$.

Miyauchi, T., Tanaka, K., Hagimoto, H., Miura, T., Kishimoto, H., Matsushita, M., 1990. Computerized EEG in schizophrenic patients. Biol. Psychiatry 28, 488-494.

Mohr, C., Blanke, O., 2005. The demystification of autoscopic phenomena: experimental propositions. Curr. Psychiatry Rep. 7, 189-195.

Mohr, C., Krummenacher, P., Landis, T., Sandor, P.S., Fathi, M., Brugger, P., 2005. Psychometric schizotypy modulates levodopa effects on lateralized lexical decision performance. J. Psychiatr. Res. 39, 241-250.
Mohr, C., Blanke, O., Brugger, P., 2006. Perceptual aberrations impair mental own-body transformations. Behav. Neurosci. 120, 528-534.

Murray, C.D., Fox, J., 2005. Dissociational body experiences: differences between respondents with and without prior out-of-body-experiences. Br. J. Psychol. 96, 441-456.

Oldfield, R.C., 1971. The assessment and analysis of handedness: the Edinburgh inventory. Neuropsychologia 9, 97-113.

Omori, M., Koshino, Y., Murata, T., Murata, I., Nishio, M., Sakamoto, K., Horie, T., Isaki, K., 1995. Quantitative EEG in never-treated schizophrenic patients. Biol. Psychiatry 38, 305-309.

Pascual-Marqui, R.D., Michel, C.M., Lehmann, D., 1995. Segmentation of brain electrical activity into microstates: model estimation and validation. IEEE Trans. Biomed. Eng. 42, 658-665.

Perrin, F., Pernier, J., Bertrand, O., Giard, M.H., Echallier, J.F., 1987. Mapping of scalp potentials by surface spline interpolation. Electroencephalogr. Clin. Neurophysiol. 66, 75-81.

Platek, S.M., Gallup Jr., G.G., 2002. Self-face recognition is affected by schizotypal personality traits. Schizophr. Res. 57, 81-85.

Pope, C.A., Kwapil, T.R., 2000. Dissociative experience in hypothetically psychosis-prone college students. J. Nerv. Ment. Dis. 188, 530-536.

Rado, S., 1960. Theory and therapy, the theory of schizotypal organization and its application to the treatment of decompensated schizotypal behavior. In: Scher, S.C., Davis, H.R. (Eds.), The Outpatient Treatment of Schizophrenia. Grune and Stratton, New York, pp. 87-101.

Roth, W.T., Cannon, E.H., 1972. Some features of the auditory evoked response in schizophrenics. Arch. Gen. Psychiatry 27, 466-471.

Roth, W.T., Pfefferbaum, A., Horvath, T.B., Kopell, B.S., 1980. P300 and reaction time in schizophrenics and controls. Prog. Brain Res. 54, 522-525.

Ruby, P., Decety, J., 2001. Effect of subjective perspective taking during simulation of action: a PET investigation of agency. Nat. Neurosci. 4, $546-550$.

Saito, N., Kuginuki, T., Yagyu, T., Kinoshita, T., Koenig, T., PascualMarqui, R.D., Kochi, K., Wackermann, J., Lehmann, D., 1998. Global, regional, and local measures of complexity of multichannel electroencephalography in acute, neuroleptic-naive, first-break schizophrenics. Biol. Psychiatry 43, 794-802.

Saxe, R., Kanwisher, N., 2003. People thinking about thinking people. The role of the temporo-parietal junction in "theory of mind". NeuroImage $19,1835-1842$

Siever, L.J., Davis, K.L., 2004. The pathophysiology of schizophrenia disorders: perspectives from the spectrum. Am. J. Psychiatry 161, 398-413.

Simeon, D., Guralnik, O., Hazlett, E.A., Spiegel-Cohen, J., Hollander, E., Buchsbaum, M.S., 2000. Feeling unreal: a PET study of depersonalization disorder. Am. J. Psychiatry 157, 1782-1788.

Spence, S.A., Brooks, D.J., Hirsch, S.R., Liddle, P.F., Meehan, J., Grasby, P.M., 1997. A PET study of voluntary movement in schizophrenic patients experiencing passivity phenomena: delusions of alien control. Brain 120, 1997-2011.

Strik, W.K., Dierks, T., Franzek, E., Stöber, G., Maurer, K., 1994. P300 assymmetries in schizophrenia revisited with reference-independent methods. Psychiatry Res. NeuroImaging 55, 153-166.

Tallent, K.A., Gooding, D.C., 1999. Working memory and Wisconsin Card Sorting Test performance in schizotypic individuals: a replication and extension. Psychiatry Res. 89, 161-170.

Vogeley, K., Fink, G.R., 2003. Neural correlates of the first-personperspective. Trends Cogn. Sci. 7, 38-42.

Zacks, J., Rypma, B., Gabrieli, J.D., Tversky, B., Glover, G.H., 1999. Imagined transformations of bodies: an fMRI investigation. Neuropsychologia 37, 1029-1040. 